\title{
Morphology of coronary arteries in relation to ischemic heart disease
}

\begin{abstract}
Background: The anatomical features of the coronary circulation show some interesting data which have been obtained by both in vivo and post-mortem studies.

Methods: This editorial describes the morphology and main alterations of coronary arteries, which supply blood flow to heart muscle, observed in the ischemic heart disease.

Results: The great majority of myocardial muscle is under the control of the left coronary arteries: the left main coronary artery and its branches, the left anterior descending artery, and circumflex artery, which provide several arterial vessels particularly to the left anterior ventricular wall, interventricular septum and apex of the heart. Therefore, a large portion of the posterior left ventricular wall is supplied by left coronary circulation. The right coronary artery supplies blood flow to the posterior wall of the heart and the right atrium. Coronary anastomoses have been demonstrated into the myocardium showing that coronary arteries widely communicate among themselves. The main lesions of the coronary tree in subjects suffering from ischemic heart disease are related to coronary atherosclerosis.
\end{abstract}

Conclusion: Morphology of coronary arteries is strongly influenced by ischemic pathology of the heart.

Keywords: coronary circulation, coronary artery, morphology, ischemic heart disease, atherosclerosis, thrombosis
Volume I4 Issue 2 - 202 I

\author{
Aurelio Leone ${ }^{1,2}$ \\ 'Department of Internal Medicine, Fellow of the Royal Society \\ for Promotion of Health, UK \\ ${ }^{2}$ Department of Internal Medicine, Fellow of the American \\ Heart Association, USA
}

Correspondence: Aurelio Leone, Department of Internal Medicine, Fellow of the Royal Society for Promotion of Health, UK, Email reliol@libero.it

Received: March 03, 202I | Published: March 31, 202 I

\section{Editorial}

Ischaemic heart disease shows a strong epidemiological impact despite of the significant progresses obtained by diagnostic and therapeutic approaches. This occurrence is a result of two main factors: the close relationship existing between epidemiology and cardiovascular risk factors and, in addition, the type of alterations affecting the coronary tree of the individuals suffering from ischemic pathology, as many findings undoubtedly show. ${ }^{1-4}$ The analysis of the anatomy of coronary tree is worthy of mention in an attempt to better understand those alterations observed in the ischaemic heart disease.

In-vivo angiography and post-mortem study of the coronary arteries using a combined method of post-mortem injection plus dissection provide those results which allow clarifying the morphology of the coronary tree..$^{5-9}$ The purpose of this editorial, which summarizes a great number of personal findings previously published, is to discuss the morphological characteristics of the coronary arteries and the relationship with ischemic heart disease.

\section{Anatomical patterns of coronary arteries}

Coronary arteries supply blood flow to the heart in both physiologic and pathologic conditions. The benchmark of the coronary circulation is providing the heart of an amount of blood volume depending on body needs, otherwise, adjusting the blood volume to metabolic demand of cardiac muscle when this parameter is changing with regard to degree of cardiac working.

Three major coronary vessels as conduit arteries (epicardial vessels) together with an intricate network of end small arteries characterize the coronary circulation.

\section{Epicardial coronary arteries and intramyocardial circulation}

The structure of epicardial and intramyocardial circulation of the coronary arteries explains possible different mechanisms of cardiovascular damage observed in the individuals suffering from ischemic heart disease. Usually, the alterations are related to hypoxia that triggers anatomical and/or functional changes in vessel structures with a lumen reduction due to isolated or multiple stenoses of different size and degree.

Physiologically, conduit vessels ${ }^{10}$ are cylindrical arteries that reduce progressively their caliber along the entire length from their origin to the end. This geometrical characteristic allows carrying out the basic function consisting of blood flow circulation.

Geometrically, a cylinder is a uniform and hollow threedimensional solid with straight sides and a circular section. It arises from a complete rotation of a side of a plane figure, a rectangle, giving a three-dimensional solid. On the contrary, resistance vessels are intramyocardial segments and their anastomoses, which play a basic role to regulate the coronary circulation. Indeed, heart function by systolic and diastolic phases influences intramyocardial coronary vessels stronger than all other biochemical metabolites. By this mechanism, it contributes to regulate coronary blood flow and intramyocardial metabolic changes. Major changes in resistance of coronary circulation are mediated primarily by those intramyocardial coronary vessels having a diameter from $10 \mathrm{~m} \mu$ to $140 \mathrm{~m} \mu .^{10,11}$ Anatomically, a specific type of gross and histologic structure characterizes conduit and resistance coronary arteries as Table 1 shows. 
Table I Morphological characteristics of coronary tree

\begin{tabular}{lll}
\hline Coronary vessel & Lumen diameter $(\mathrm{mm})$ & Myocardial distribution \\
\hline Conduit artery vessels & $4.2+/-0.6$ to $2.8+/-0.5$ & $\begin{array}{l}\text { Epicardial arteries and first } \\
\text { intramyocardial }\end{array}$ \\
Resistance artery vessels & 140 to $10 \mathrm{mcr}$ & $\begin{array}{l}\text { Intramyocardial coronary arteries, } \\
\text { anastomoses } \\
\text { intercoronary and intracoronary } \\
\text { anastomoses })\end{array}$ \\
\hline
\end{tabular}

\section{Epicardial coronary arteries}

Two major coronary arteries, which arise from the sinus of Valsalva behind the aortic valve leaflets, and their branches, provide blood flow to the heart. The two main coronary arteries are known as left main coronary artery and right coronary artery. Inconstantly, a third coronary artery, named conus artery, originates from a separate ostium in the right sinus in about 50 percent of individuals. Additional small ostia have been described to be into the right sinus, but with a poor role in regulating coronary blood flow.

The left main coronary artery, starting from the left posterior sinus of Valsalva, goes between the pulmonary artery and the left auricular appendix. After a short course usually varying from $0.5 \mathrm{~cm}$. to $2 \mathrm{~cm}$. It branches, usually, into two vessels, which are known respectively as the anterior descending artery, the main coronary artery, and the circumflex artery. Left main coronary artery may branch in 60 percent of cases ${ }^{12}$ in three or, very rarely, four small arteries. Among these, it is worthwhile mentioning the diagonal artery because it supplies an important part of the marginal myocardium. The right coronary artery branches more distally about its origin compared to what characterizes left main coronary artery. Functionally, the coronary arteries were believed to be, in the past, end-arteries, although anatomically, numerous intercoronary and intracoronary (or homocoronary) anastomoses up to $40 \mu \mathrm{m}$ in diameter, differently filled, in most normal hearts exists.

The left and right coronary arteries supply different myocardial areas, and the knowledge of blood flow distribution explains both the localization and course of myocardial alterations as well as the relationship between the type and extent of the vascular lesion, which are associated to the ischemic heart disease.

The anterior descending artery goes vertically 1 to $1.5 \mathrm{~cm}$ from the left main coronary artery. It courses down into the anterior interventricular groove, reaches the anterior portion of the cardiac apex rounding the acute margin of the heart and, then, goes up the posterior interventricular groove for about $1 \mathrm{~cm}$. The left coronary artery supplies (Table 2) particularly the anterior wall of the left ventricle, the bordering third of the anterior surface of the right ventricle, the anterior two-thirds of the interventricular septum and most of the heart apex. Therefore, it extends the largest mass of the left myocardium feeling maximally systolic and diastolic effects of heart function. Along its course left anterior descending artery gives off, in an usual order of origin, the first diagonal coronary artery, that can arise, as it will be described, from the left main coronary artery, the first septal perforator branch, and three to four septal perforator arteries as well as some other diagonal branches.

Table 2 Myocardial areas supplied by the coronary arteries, both conduit and resistance vessels

\begin{tabular}{|c|c|c|}
\hline Coronary artery & Caliper $(\mathrm{mm}) *$ & Myocardial area supplied \\
\hline Anterior descending coronary artery & $4.5+/-0.5$ & $\begin{array}{l}\text { Anterior wall of the left ventricle } \\
\text { Border third of the anterior surface of right ventricle }\end{array}$ \\
\hline Right coronary artery & $3.9+/-06$ & $\begin{array}{l}\text { Anterior and posterior right ventricular wall } \\
\text { Posterior third of left ventricular wall } \\
\text { Posterior third of interventricular septum }\end{array}$ \\
\hline Circumflex coronary artery & $3.7+/-0.4$ & $\begin{array}{l}\text { Small portions of lateral wall of the left ventricle } \\
\text { Small portion of left ventricle anterior wall } \\
\text { Small portion of left ventricle posterior wall }\end{array}$ \\
\hline Diagonal artery & & $\begin{array}{l}\text { Interventricular septum } \\
\text { Anterior wall of the left ventricle } \\
\text { Anterior papillary muscle }\end{array}$ \\
\hline
\end{tabular}

*Measures of the epicardial first course

The right coronary artery (Table 2), after the origin from the right anterior Valsava sinus of the aorta, courses the right atrioventricular sulcus, rounds the acute margin reaching the crux in a large majority of subjects and, then, provides a variable number of branches distributed in the anterior right ventricular wall. A branch (marginal branch), usually, courses the acute margin of the heart, while another branch, named posterior interventricular descending artery, courses down into the posterior interventricular groove reaching the posterior portion of the apex to about $1 \mathrm{~cm}$. above. Along its course the right coronary artery provides a small branch to supply atrioventricular node as well as branches originating shortly after its take-off, to reach atrial myocardium and sinoatrial nodus. By this way, the right coronary artery supplies the reminder wall of the right ventricle anteriorly and posteriorly, the posterior third of both left ventricular wall and interventricular septum and most of atrial myocardium. Moreover, a little branch (infundibular coronary artery) arises from this vessel to ramify into the anterior portion of the arterious conus of the right ventricle.

The circumflex artery (Table 2), usually a smaller branch if compared to the left anterior descending artery arising from the left main coronary artery, runs in the left ventricular sulcus after its origin and supplies a small portion of the lateral myocardium of the left 
ventricle together with a small portion of the left ventricle anteriorly and posteriorly and left atrium by giving small branches in these areas. Usually, the circumflex artery ends at the obtuse, margin of the heart, although, in some occurrences, it can reach the crux - namely the junction of the posterior interventricular sulcus and posterior atrioventricular groove-. When this anatomical distribution occurs, all the left ventricle and ventricular septum are supplied by blood of the left coronary arteries relieving the flow into the right coronary artery.

Finally, the diagonal artery that, usually, rises near the bifurcation of the left main coronary artery, courses inferiorly along an angle of the anterior wall of the left ventricle initially between the circumflex and the left anterior descending artery and, then, between the left descending artery and obtuse marginal artery. The diagonal artery supplies little branches to the interventricular septum, the anterior wall of the left ventricle, and anterior papillary muscle.

A Subepicardial portion of the coronary arteries is embedded in a various amount of subepicardial fat up their entering into myocardial mass. An excellent and complete description of the anatomy of coronary arteries may be examined in some recent reports. ${ }^{13,14}$ specifically deputed to this topic.

Ii is worth noting that starting from these data there is evidence that variations in branching patterns may be seen in the human heart, making dominant, sometimes, the right coronary circulation, sometimes the left coronary circulation. Otherwise, a balanced coronary circulation, according to the type and distribution of the coronary tree, is usually documented. Different locations of lesions may be influenced by the characteristics and distribution of the coronary tree.

\section{Intramyocardial circulation}

Intramyocardial circulation (Table 3 ) consists of: 1. Intramyocardial coronary arteries; 2. Coronary arterioles; 3 . Intercoronary and intracoronary (homocoronary) anastomoses; 4 . Capillary bed.

Table 3 Type of intramyocardial coronary artery vessels

Large intramyocardial coronary arteries (all major
coronary vessels)
Small intramyocardial coronary arteries
Intramyocardial coronary arterioles
Intercoronary anastomoses
Homocoronary anastomoses
Capillary bed

Coronary vessels enter the myocardial mass perpendicularly. They have different length and caliber, which diminishes progressively from myocardial wall, entering to branching in the capillary network. The Table 3 shows type and the distribution of the intramyocardial artery vessels. There is evidence of an increasing reduction in the caliper of the resistance arteries along their course.

Intramural coronary circulation feels deeply either the anatomical integrity of extramural coronary arteries or myocardial mass since these vessels are respectively stressed and released during systole and diastole of the cardiac cycle. In case of a poor availability of oxygen, intramyocardial vessels suffer chronically and show, in time, hyperplastic and degenerative changes characterized by impairment of the metabolic function. ${ }^{14,15}$

Anastomoses, which may be observed also in normal hearts, clearly show that the coronary arteries are not end-arteries, as believed in the past, but they form a network structurally organized in any intramyocardial area. Moreover, anastomoses, independently on the development of coronary collaterals, may be seen at any level of myocardial mass, although the maximum development has been demonstrated to occur distally.

\section{Geometrical characteristics of coronary tree}

Techniques of study carried out by using post-mortem coronary injection with a radiopaque mass associated with a chemical digestion of myocardial mass permitted to obtain heart casts which give threedimensional pictures of the coronary tree and, in addition, permit to identify cardiac alterations of both heart and coronary vessels (Figures $1-3)$.

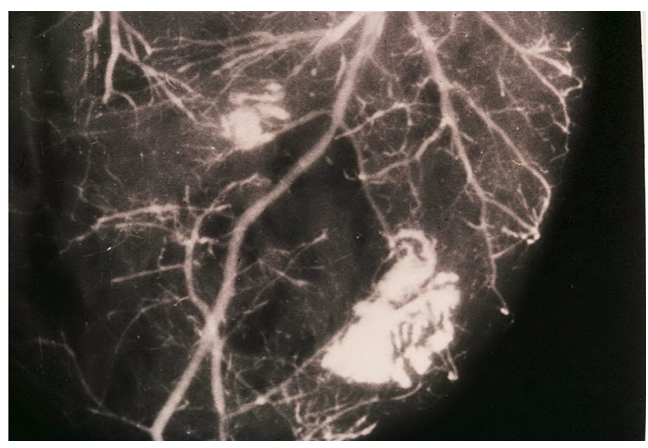

Figure I Post-mortem angiography obtained by using barium sulfate combined with bacto-gelatin of a part of myocardial mass in a heart cast. There is evidence of coronary collaterals, intercoronary and intracoronary anastomoses as well as an avascular area in the central zone of the left ventricular wall due to an old myocardial infarction (white arrows). Reproduced from Aurelio Leone: ${ }^{13}$ Anatomy of the Coronary Arteries.

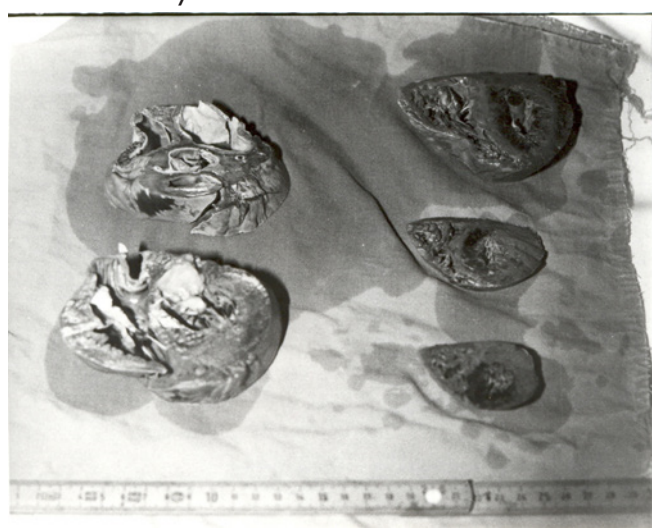

Figure 2 Gross study of the heart following post-mortem coronary angiography. Cardiac muscle cut in parallel slices of about $\mathrm{I} \mathrm{cm}$ of thickness permits to identify areas of heart damage.

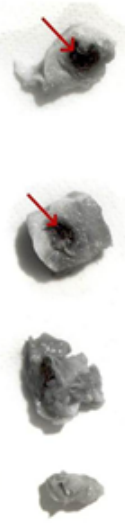

Figure 3 Gross study of left descending coronary artery showing an occlusive thrombus (red arrow) in a subject died from acute anterior myocardial infarction. 
Briefly, the post-mortem method consists of coronary injection, after removing the heart. ${ }^{6,8,15}$ by severing the pulmonary artery and aorta about $5 \mathrm{~cm}$ from the free margin of the semilunar valves. After observing the external aspect of the heart to identify existing areas of myocardial damage, without opening the coronary vessels, a plug is placed through the aorta into the aortic orifice and then the coronary arteries are injected at a pressure of $130 \mathrm{mmHg}$ using a barium-iodinegelatin radiopaque mass by a cannula tied into the aorta. ${ }^{6}$ When a good degree of contrast is noted on $\mathrm{x}$-rays, the heart undergoes cast building by a chemical procedure. ${ }^{12} \mathrm{~A}$ direct injection of radiopaque mass of each single coronary artery through a cannula into its orifice of origin may be also used. ${ }^{15,20}$ After the injection, maceration and corrosion of the heart a vascular cast is obtained. Customarily, the injection mass must be of material that resists to corrosive techniques. Myocardial mass is destroyed by strong alkaline or acid solutions.

Casts permitted to identify primarily some spatial (threedimensional) characteristics of the coronary artery system that could better explain the relationship existing between coronary tree and blood flow supply.

Firstly, it is worth noting that numerous anastomoses, which differ with regard to type, caliber, and distribution, may be documented into intramyocardial mass. These anastomoses occur between branches of the same coronary artery as intracoronary or homocoronary anastomoses and as branches of different intramyocardial coronary arteries. Anastomoses show to be either functioning structures or empty structures in relation to the metabolic demand of the heart. Therefore, they may be observed transiently or, on the contrary, permanently, but they exist with no doubt.

Secondly, the course of the main subepicardial coronary arteries may be carefully established. The left coronary tree, that arise from the posterior aortic ostium, goes anteriorly towards the anterior wall of the left ventricle, while the right coronary artery from the anterior aortic ostium reaches the posterior wall of the left ventricle. This running is in relation to the portion of the heart that coronary tree supplies.

Finally, not yet well demonstrated metabolic responses under stress, risk factors and heavy coronary pathology determine the development of the coronary collateral circulation in a great number of ischemic patients. ${ }^{16}$ Usually, the development of coronary collaterals is related to significantly chronic coronary artery pathology ${ }^{17}$ in individuals affected from ischemic heart disease.

Nowadays, post-mortem coronary injection of radiopaque mass and casts to identify the morphology of the coronary arteries are used even less because of the development of refined instrumental techniques of study in vivo like three-dimensional echocardiography, computed tomography and nuclear magnetic resonance, which permit to obtain selective images of the different cardiac structures totally similar to those observed by necropsy examination. Thus, the most updated diagnostic and instrumental techniques are used even more frequently because of the progress in therapeutic treatment of coronary artery disease in those patients who are at risk.

\section{Histology of coronary arteries}

Likewise, other arteries of the body, coronary wall in conduit vessels, namely epicardial coronary vessels and large intramural coronary vessels, consists of three coats differently developed: tunica intima, tunica media, and tunica adventitia.

The intima, the inner lamina layer, consists of a lining of endothelial cells, and a subendothelial layer where there are connective tissue and smooth muscle cells. All these structures characterize the endothelium, which is a selective barrier of diffusion between the blood and other wall coats. Spatially, endothelial cells are oriented longitudinally along the vessel and attached between them by junctions of two types: occluding junctions and gap junctions.

Over the last twenty years, experimental and clinical results experienced that the endothelium is a widely distributed organ among and within tissues ${ }^{18,19}$ deeply involved to regulate vascular physiology and the pathophysiologic changes. These functions are closely related to the position at the interface between blood and tissue and physiologically are regulated by vasodilator and vasoconstrictor metabolites. ${ }^{20-22}$

The intima is separated from the medial coat by the internal elastic line, which may be differently structured in relation to its integrity or harm due to injuring factors.

Medial coat consists of multiple layers of smooth muscle cells, collagen and elastic fibers. There is evidence that the amount of each of the above components varies widely with regard to coronary course, being elastic material less and smooth muscle cells greater in the epicardial coronary arteries compared with elastic arteries of different locations of the body. ${ }^{23}$

Spatially, the media may consist of up 40 layers of smooth muscle cells oriented in a circle or helix and embedded in a connective glycoprotein mix.

An external elastic lamina, considerably thinner than the internal elastic lamina and composed particularly of elastin, separates medial from adventitial coat.

Medial coat is the structure which feels more deeply the pathologic injures due to the effects of the major coronary risk factors.

Adventitial coat consists ${ }^{12}$ of a significant amount of fibrous tissue, particularly collagen and elastic fibers oriented longitudinally. In its context vasa vasorum, lymphatic vessels and nerves exist. The thickness of the coat may vary widely going from 300 to $500 \mu \mathrm{m}$.

From the origin to intramyocardial coronary network, there is a progressive reduction of the thickness of adventitial and medial coats whereas endothelium improves its metabolic and functional properties.

\section{Ultrastructural morphology of coronary arteries}

At the electron microscopy, the structure of epicardial coronary arteries does not differ to that of all other arteries. However, vascular endothelium of the coronary tree is most frequently injured by the effects of the major risk factors, which can cause, usually, endothelial dysfunction..$^{24,25}$

It is worth noting that electron microscopy shows a great number of basic constituents of the coronary wall as the endothelial cells, smooth muscle cells together with an extracellular matrix particularly composed by connective material including elastic fibers, collagen and proteoglycans. Usually, the intima shows polygonal cells, which are in contact with arterial lumen. Minimal underlying subendothelial connective tissue is also present. Media shows particularly smooth muscle cells circularly or spirally oriented. Elastin, the one of the major components, is usually arranged near the internal and external elastic lamina. Nervous components and metabolic granules of vasoactive substances are present. Finally, a great number of elements belonging to connective tissue characterize the adventitia where vasa vasorum and nerve fibers are dispersed. 


\section{Characteristics of coronary lesions of ischemic heart disease}

Nearly totally accepted opinion is coronary atherosclerosis has been demonstrated the strongest factor of development of coronary artery wall lesions primarily when cardiovascular risk factors may be identified in those subjects suffering from ischemic heart disease. ${ }^{26}$ In addition, evidence indicates that coronary lesions can be of minimal up to severe degree. ${ }^{27-30}$

The most dramatic events ${ }^{31-35}$ are the coronary plaque rupture and hemorrhage as well as thrombus totally occluding the coronary lumen primarily in those patients where coronary collateral circulation is not yet completely developed. The last outcome is strongly related to morphopathology of coronary circulation while atherosclerotic plaque rupture particularly feels the metabolic characteristics of the individual. It is worth noting that atherosclerotic plaque ${ }^{36}$ is primarily composed of lipids, inflammatory cells and fibrotic material. ${ }^{36-39}$ Both types of lesions have been associated to nonfatal and fatal acute myocardial infarction as well as sudden death.

These observations, although timely dated, are almost universally accepted to be the basis explaining the mechanisms responsible of cardiac ischemic pathology.

A great number of lesions of the coronary tree, listed in the Table 4, accompany the individuals who suffer from cardiac ischemic pathology.

Table 4 Coronary lesions of the ischemic heart disease

\begin{tabular}{ll}
\hline Coronary Alteration & Morphopathology \\
\hline \multirow{2}{*}{ Minimal coronary lesion } & Artery wall fibrosis \\
& Wall thickening \\
& Sub-occlusive thrombi \\
& Coronary plaque \\
& Stable \\
Coronary lumen reduction (narrowings) & Regression \\
& Vulnerable \\
& Rupture \\
& Hemorrhage \\
& Embolism \\
& Occlusive thrombi \\
Coronary lumen totally occluded & Partially or totally combined \\
& coronary lesions \\
\hline
\end{tabular}

It is worth noting that a great number of factors may influence the coronary artery morphology. Apart coronary risk factors, which play an always more documented effect in inducing coronary alterations, there are some physiologic parameters like sex, age, and lifestyle $\mathrm{e}^{40-42}$ which may change the characteristics of the coronary tree.

Commonly, the most frequent alterations of the coronary tree of ischemic heart disease consist of a narrowing of different degree. However, alterations superimposed in a different time can continuously modify the morphology of the coronary arteries. In addition, atherosclerotic plaque shows to be the main pathological structure documented in the coronary tree of the individuals suffering from an ischemic cardiac pathology. It is also able to strongly influence the appearance of symptoms and the course of heart disease. ${ }^{43}$ However, this statement is not clearly demonstrated still needing further investigations.

\section{Conclusion}

The patterns described clearly identify a strong relation between morphology of coronary arteries and the development of the ischemic heart disease. In addition, evidence indicates that the heart is, usually, more often heavily affected when coronary lesions are severe and widely diffused.

In humans, some pathological lesions of the coronary arteries are most frequently associated to acute myocardial infarction and/or sudden cardiac death; although still there is no certainty to know when these dramatic events may occur.

\section{Conflicts of interest}

The authors declare no conflict of interest, financial or otherwise.

\section{Acknowledgments}

Declared none.

\section{References}

1. Leone A. Cardiovascular damage from smoking:a fact or belief? Int $J$ Cardiol. 1993;38:113-117.

2. Hammond EC, Garfinkel L. Coronary heart disease, stroke and aortic aneurysm. Arch Environ Health. 1969;19(2):167-182.

3. Auerbach O, Carter HW, Garfinkel M, et al. Cigarette smoking and coronary heart disease, a macroscopic and microscopic study. Chest. 1976;70(6):697-705.

4. Leone A, Lopez M. Oral contraception, ovarian disorders and tobacco in myocardial infarction of woman. Pathologica. 1986;78:237-242.

5. Levin DC, Fallon JT. Significance of the angiographic morphology of localized coronary stenoses: Histopathologic correlations. Circulation. 1982;66 (2):316-320.

6. Leone A. Postmortem coronary angiography in the pathological study of the heart. G Ital Cardiol. 1972;2:688-692.

7. Roberts WC. Coronary arteries in fatal acute myocardial infarction. Circulation. 1972;45(1):215-230.

8. Baroldi G, Mantero O, Scomazzoni G. The collaterals of the coronary arteries in normal and pathological hearts. Circ Res. 1956;4(2):223-229.

9. Leone A. Passive Smoking, Endothelial Dysfunction and Related Markers. An Update. 2012;8(2):141-150.

10. Leone A, Landini L. Vascular Pathology from smoking:look at the microcirculation. Curr Vasc Pharmacol. 2013;11(4):524-530.

11. Dodge TJ, Brown J, Bolson EL, et al.. Lumen diameter of normal human coronary arteries. Influence of age, sex, anatomic variation, and left ventricular hypertrophy or dilation. Circulation. 1991;86:232-246.

12. Baroldi G. Diseases of the coronary arteries. In: Silver, editor Cardiovascular Pathology, 1. York, USA: Churchill Livingstone; 1983. p. 317-391.

13. Leone A. Anatomy of the coronary arteries. In:Coronary Circulation in Nonsmokers and Smokers, A Leone editor. USA, New York: Nova Biochemical Books; 2008; p. 1-20.

14. Nellis SH, Liedtke AJ, Whitessel L. Small coronary vessel pressure and diameter in an intact beating rabbit heart using fixed-position and freemotion techniques. Circ Res. 1981;49:342-353.

15. Schlesinger MJ. An injection plus dissection study of coronary artery occlusions and an anastomosis. Am Heart J. 1938;15(5):528-568.

16. Van der Ghinst M. Injection of the coronary system with plastics. Acta Cardiol. 1949;4:274-279.

17. Gregg DE, Patterson RE. Functional importance of the coronary collaterals. N Engl J Med. 1980;303(24):1404-1406.

18. Fishman AP. Endothelium: a distributed organ of diverse capabilities. Ann NY Acad Sci. 1982;401:1-8. 
19. Cines DB, Pollak ES, Buck CA, et al. Endothelial cells in physiology and in the pathophysiology of vascular disorders. Blood. 1998;91(10):35273561 .

20. Celermajer DS, Sorensen KE, Georgakopoulos D. Cigarette smoking is associated with dose-related and potentially reversible impairment of endothelium-dependent dilation in healthy young adults. Circulation. 1993;88:2149-2155.

21. Deedwania PC. Endothelium: a new target for cardiovascular therapeutics. J Am Coll Cardiol. 2000;35(10:67-70.

22. Panza JA, Garcia CE, Kilcoyne CM, et al. Impaired endotheliumdependent vasodilation in patients with essential hypertension. Evidence that nitric-oxide abnormality is not localized to a single transduction pathway. Circulation. 1995;91(6):1732-1738.

23. Wong $\mathrm{M}$, Edelstein $\mathrm{J}$, Wollman $\mathrm{J}$, et al. Ultrasonic-pathological comparison of the human arterial wall. Verification of intima-media thickness. Arterioscl Thromb. 1993;13(4):482-486.

24. Leone A. The Results of the Clinical Trials in Hypertension: Unquestionable Benefits and Limits. J Cardiol Curr Res. 2017. 9(3):00327.

25. Fishmam AP. Endothelium: a distributed organ of diverse capabilities Ann NY Acad Sci. 1982;401:1-8.

26. Leone A, Landini L. Modifying Cardiovascular Risk Factors:Acquired Topics and Emerging Concepts. Curr Pharm Des. 2017;23(22):31973199 .

27. Eliot RS, Baroldi G, Leone A. Necropsy studies in myocardial infarction infarction with minimal or no coronary occlusion due to atherosclerosis. Circulation. 1974;49 (86):1127-1131

28. Baroldi G, Radice F, Schimd G, et al. Morphology of acute myocardial infarction in relation to coronary thrombosis. Am Heart J. 1974;87 (1):6575 .

29. Maddox TM, Stanilawski MA, Grunwald GK, et al. Nonobstructive coronary artery disease and risk of myocardial infarction. JAMA. 2014;312 (17):1754-1763.

30. Burke AP, Farb A, Malcom JT, et al. Plaque rupture and sudden death related to exertion in men with coronary artery disease. JAMA. 1999;281 (10):921-926

31. Richardson PD, Davies MJ, Born GV. Influence of plaque configuration and stress distribution on fissuring of coronary atherosclerotic plaques. Lancet. 1989;2(8669):941-944.
32. Farb A, Burke AP, Tang AL, et al. Coronary plaque erosion without rupture into a lipid core. A frequent cause of coronary thrombosis in sudden coronary death. Circulation. 1996;93(7):1354-1363.

33. Chen L, Crook JR, Tousoulis D, et al. Complex stenosis morphology predicts late reocclusion during follow-up after myocardial infarction in patients with patent infarct related coronary arteries. Am Heart $J$ 1998;136(5):877-883.

34. Leone A. Relation between coronary lesions and cigarette smoking of subjects deceased from acute myocardial infarction. A histopathological study. J Cardiobiol. 2014;2 (2):1-5.

35. Stary HC, Chandler AB, Dinsmore RE, et al. A definition of advanced types of atherosclerotic lesions and a histological classification of atherosclerosis. A report from the Committee of Vascular Lesions of the Council on Atherosclerosis, American Heart Association. Circulation. 1995;92:1355-1374.

36. Tabas I. Macrophage death and defective inflammation resolution in atherosclerosis. Nat Rev Immunol. 2010;10(1):36-46.

37. Steinberg D. The LDL modification hypothesis of atherogenesis: an update. J Lipid Res. 2009;50 (Suppl):S376-S381.

38. Stary HC. Lipid and macrophage accumulation in arteries of children and the development of atherosclerosis. Am JClin Nutr. 2000;72:1297S-1306S.

39. Falk E, Shah PK, Fuster V. Coronary plaque disruption. Circulation. 1995;92:657-671

40. Bittner V. Menopause, age, and cardiovascular risk:a complex relationship. J Am Coll Cardiol. 2009;54(25):2374-2375.

41. Matthews KA, Crawford SL, Chae CU, et al. Are changes in cardiovascular disease risk factors in midlife women due to chronological aging or to the menopausal transition? J Am Coll Cardiol. 2009;54(25):2366-2373.

42. Kok HS, van Asselt KM, van der Schouw YT, et al. Heart disease risk determines menopausal age rather than the reverse. J Am Coll Cardiol. 2006;47(100:1976-1983.

43. Nabel EG, Braunwald E. A tale of coronary artery disease and myocardial infarction. N Engl J Med. 2012;366(1):54-63. 